\title{
Conformal Mapping and Bipolar Coordinate for Eccentric Laplace Problems
}

\author{
JENG-TZONG CHEN, ${ }^{1,2}$ MING-HONG TSAI, ${ }^{1}$ CHEIN-SHAN LIU ${ }^{2}$ \\ ${ }^{1}$ Department of Harbor and River Engineering, National Taiwan Ocean University, Keelung, Taiwan \\ ${ }^{2}$ Department of Mechanical and Mechatronic Engineering, National Taiwan Ocean University, Keelung, Taiwan
}

Received 28 July 2008; accepted 5 October 2008

\begin{abstract}
Boundary value problems on the eccentric annulus are quite complex and cannot directly be solved analytically using cartesian or polar coordinates. Many mathematical techniques have been used to solve such a problem by using conformal mapping and bipolar coordinate. In the literature, Carrier and Pearson [Partial differential equation-theory and technique. New York, Academic Press, 1976, pp 68-71], Muskhelishvili [Some basic problems of the mathematical theory of elasticity. Noordhoff, Groningen; 1953, pp 175-179], Ling [Torsion of an eccentric circular tube, Technical Report, No. 1, Chinese Bureau of Aeronautical Research, 1940], Timoshenko and Goordier [Theory of Elasticity. New York, McGraw-Hill; 1972, pp 196-202], Shen [Null-field approach for Laplace problems with circular boundaries using degenerate kernels, Master thesis, National Taiwan Ocean University, Keelung, Taiwan, 2005], Lebedev et al. [Worked Problems in Applied Mathematics. New York, Dover; 1965] have solved this kind of problems using similar techniques. By using transformation in a transformed plane in the complex variable theory, we can obtain the analytical solution easily. We focus on the connection between conformal mapping and curvilinear coordinates, and figure out the relation to take integration by way of mapping in the complex plane. All the transformations and curvilinear coordinates can be unified using the viewpoint of conformal mapping. Their relationship among available methods can be constructed by translation, stretching, rotation and inversion. Finally, an example of eccentric domain is solved by using various mappings and curvilinear coordinates and their relations are linked. Not only geometry transformation is addressed but also the solution of the Laplace equation is obtained. (c) 2009 Wiley Periodicals, Inc. Comput Appl Eng Educ 17: 314-322, 2009; Published online in Wiley InterScience (www.interscience.wiley.com); DOI 10.1002/cae.20208
\end{abstract}

Keywords: conformal mapping; bipolar coordinate; complex plane; transformation; eccentric circle; Laplace equation

Correspondence to J.-T. Chen (jtchen@mail.ntou.edu.tw). (C) 2009 Wiley Periodicals Inc.

\section{INTRODUCTION}

Based on the complex variable theory, a number of physical problems governed by Laplace equation in 
two independent variables, for example, steady-state heat condition, electrostatic potential and fluid flow, can be solved by using conformal mapping to obtain an analytical solution. Also we can formulate the same problems using different curvilinear coordinates to obtain a solution, for example, bipolar coordinate and elliptic coordinate. Carrier and Pearson [1] used a technique for solving certain kinds of potential problems, based on the bilinear transformation of conformal mapping. The eccentric case was mapped to an annular domain. For a polygonal shape, it can also be mapped to regular region by using the Schwarz-Christoffel transformation [2,3]. For the regular domain, it is easy to solve the Laplace equation subject to Dirichlet boundary condition in the polar or rectangular coordinate. Muskhelishvili [4] gave us a detailed description how an eccentric annulus can be mapped into concentric annulus using a simple form of linear fractional transformation. Shen [5], and Chen and Weng [7] also used the same method to solve eccentric annulus problems. Although a bilinear transformation was used, the mapping functions were not exactly the same between the one of Carrier and Pearson [1] and that of Muskhelishvili [4]. Some differences such as translation and stretching can be found $[6,10]$. Problems involving two nonconcentric boundaries usually require the use of the bipolar coordinate. Ling [8], Timoshenko and Goordier [9], and Lebedev et al. [11] all presented an analytic solution using the bipolar coordinate for the torsion of an eccentric bar. However, the mapping functions were also not exactly the same. One [8] is a cotangent function, another [11] is a hyperbolic tangent function and the other [9] is a hyperbolic cotangent function. After the bipolar coordinate is introduced, the special domain problem can be solved by using separation of variable in a regular domain. A typical example would be the electric field surrounding two parallel cylindrical conductors. Stephens and Casemore [12], proposed an alternative method to determine the negative stiffness coefficients for a large class of magnetic actuators. They solved the Dirichlet Laplace problems for the magnetomotive force in the actuator air gap, subject to periodic boundary conditions that can be represented by Fourier series. We can find that the bipolar coordinate is actually a kind of conformal mapping. Although Carrier and Pearson [1], Muskhelishvili [4], Ling [8], Timoshenko and Goordier [9], Shen [5], Stephens and Casemore [12] have solved the eccentric Laplace problems, their approaches are very similar but they are not fully equivalent.

This paper will link the relationship of various approaches from the viewpoint of conformal mapping.
The other parts of the present paper are arranged as follows. In Conformal Mapping Using Bilinear Function Section, the method of conformal mapping is reviewed and the analytic solution of the Laplace equation with an annular domain is reviewed. In Geometric Characterization of the Bipolar Coordinate Section, we not only describe the geometric characterization of bipolar coordinate but also derive the analytic solution of Laplace's equation. In An Illustrative Example Section, an example is demonstrated to link the relationship among many previous approaches based on the viewpoint of conformal transformation through translation, stretching, rotation and inversion. Finally, a conclusion is drawn in the fifth section.

\section{CONFORMAL MAPPING USING BILINEAR FUNCTION}

Definition of the bilinear transformation is given below:

$$
z=\frac{a w+b}{c w+d}
$$

where $a, b, c, d$ are constants (in general, complex). Equation (1) is also called a linear fractional transformation or Möbius transformation [2]. Solving (1) for $w$ in terms of $z$, we have, likewise bilinear, the inverse transformation:

$$
w=\frac{-d z+b}{c z-a}
$$

The bilinear transformation has a remarkable property that circles map to circles. The equation of any circle in the $z$ plane is known to be of the following form:

$$
A\left(x^{2}+y^{2}\right)+B x+C y+D=0
$$

where $A, B, C$, and $D$ are real constants. Since $x=((z+\bar{z}) / 2), y=((z-\bar{z}) / 2 i), x^{2}+y^{2}=z \bar{z}$, Equation (3) can be reformulated to:

$$
A z \bar{z}+M z+\bar{M} \bar{z}+D=0
$$

where $M$ and $\bar{M}$ are conjugate complex constants. By representing $z$ in terms of $w$ using Equation (1) and substituting into Equation (4), we have

$$
A_{0} w \bar{w}+M_{0} w+\bar{M}_{0} \bar{w}+D_{0}=0
$$

where $A_{0}$ and $D_{0}$ are real, and $M_{0}$ and $\bar{M}_{0}$ are conjugate complex constants. This confirms that a circle in the $z$ plane (Eq. 3) implies another circle in the $w$ plane (Eq. 5). This provides one way to map an 
eccentric case to an annulus case. For an eccentric annulus, a simple bilinear transformation will be studied here, as shown below:

$$
z=\frac{w}{1-a w}
$$

where $z=x+i y, w=\rho \mathrm{e}^{i \phi}$ and $a$ is a real positive constant. We consider now two circles $L_{1}$ and $L_{2}$ in the $z$ plane, which can be mapped to the two circles $L_{1}^{\prime}$ and $L_{2}^{\prime}$ with radii $\rho_{1}$ and $\rho_{2}$ in the $w$ plane (Fig. 1). From Figure 1, we can find the circle $L^{\prime}$ in the $w$ plane with the radius $\rho$. For the mapped annular case, we have $\rho_{1}$ and $\rho_{2}$ for inner and outer radii, respectively. Based on Equations (3) and (5), we can determine the origins and radii of $L_{1}$ and $L_{2}$ in the $z$ plane as shown below:

$$
\begin{aligned}
c_{i}=\frac{a \rho_{i}^{2}}{1-a^{2} \rho_{i}^{2}}, & i=1,2 \\
r_{i}=\frac{\rho_{i}}{1-a^{2} \rho_{i}^{2}}, & i=1,2
\end{aligned}
$$

The complex function maps eccentric circles with radii of $r_{1}$ and $r_{2}$ in the $z$ plane onto the annular region bounded by the inner radius of $\rho_{1}$ and outer radius of $\rho_{2}$ in the $w$ plane as shown in Figure 1 where $l$ is the distance between their centers. Therefore it is easy to determine the quantity $a$, appearing in (6), and the radii $\rho_{1}, \rho_{2}$ of the circles $L_{1}^{\prime}$ and $L_{2}^{\prime}$. In real practice, three quantities $r_{1}, r_{2}$, and $l$ are given in the $z$ plane. For the $w$ plane, $\rho_{1}, \rho_{2}$, and $a$ satisfy the following three equations:

$$
r_{1}=\frac{\rho_{1}}{1-a^{2} \rho_{1}^{2}}
$$

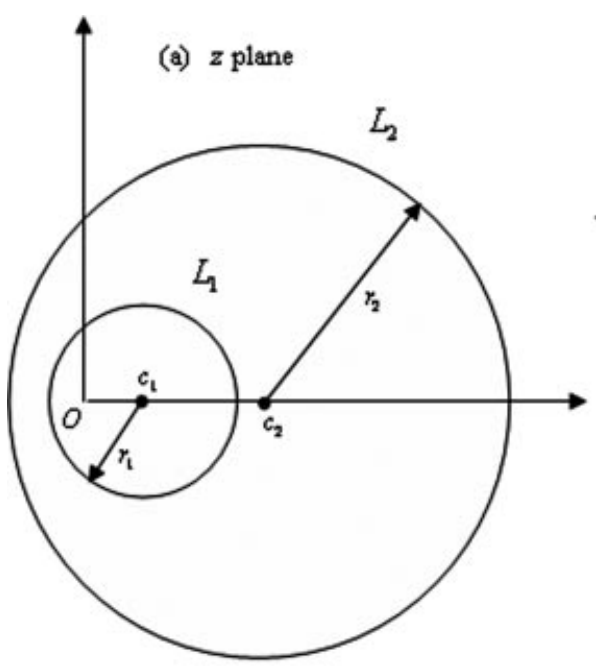

$$
\begin{gathered}
r_{2}=\frac{\rho_{2}}{1-a^{2} \rho_{2}^{2}} \\
l=c_{2}-c_{1}=\frac{a \rho_{2}^{2}}{1-a^{2} \rho_{2}^{2}}-\frac{a \rho_{1}^{2}}{1-a^{2} \rho_{1}^{2}}
\end{gathered}
$$

According to three equations $(9-11)$, three unknown constants $\rho_{1}, \rho_{2}$, and $a$ can be determined by

$$
\begin{gathered}
a=\frac{l}{\sqrt{\left(r_{1}^{2}-r_{2}^{2}\right)^{2}-2 l^{2}\left(r_{1}^{2}+r_{2}^{2}\right)+l^{4}}} \\
\rho_{1}=\frac{\sqrt{1+4 r_{1}^{2} a^{2}}-1}{2 r_{1} a^{2}}, \quad \rho_{2}=\frac{\sqrt{1+4 r_{2}^{2} a^{2}}-1}{2 r_{2} a^{2}}
\end{gathered}
$$

Then we can obtain an annulus with the radii of $\rho_{1}$ and $\rho_{2}$ from any eccentric annulus using mapping of Equation (6) with an appropriate value of $a$.

\section{Derivation of an Analytical Solution for the Laplace Equation With an Annular Domain}

By using the bilinear transformation, an eccentric domain can be mapped to an annulus domain. The Dirichlet problem for an annular domain can be described as shown below:

$$
\begin{gathered}
\nabla^{2} u=\frac{\partial^{2} u}{\partial r^{2}}+\frac{1}{r} \frac{\partial u}{\partial r}+\frac{1}{r^{2}} \frac{\partial^{2} u}{\partial \theta}=0, r_{1}<r<r_{2} \\
u\left(r_{1}, \theta\right)=f_{1}(\theta), u\left(r_{2}, \theta\right)=f_{2}(\theta)
\end{gathered}
$$

In addition, $u(r, \theta)$ must satisfy the periodicity condition. Accordingly, $f_{1}(\theta)$ and $f_{2}(\theta)$ must also be periodic with a period of $2 \pi$. Thus, the general solution is

\section{(b) $w$ plane}

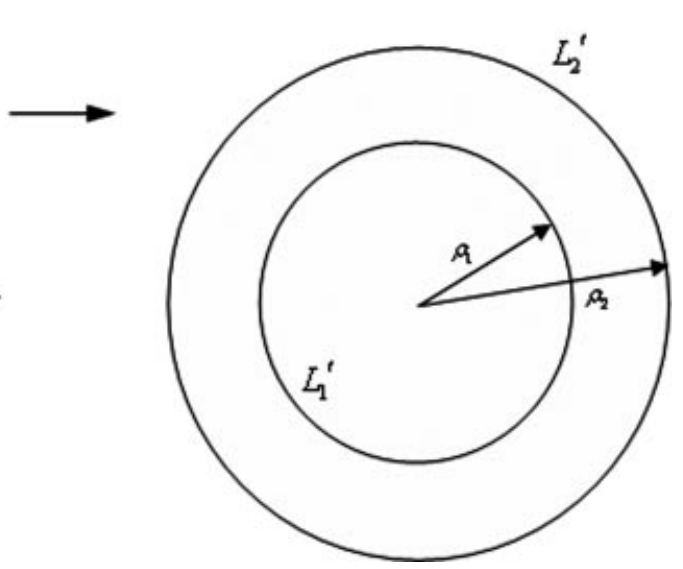

Figure 1 Mapping of $w(z)=\frac{z+\frac{1}{4}}{z+4}$ from the eccentric region to a concentric region. 


$$
\begin{aligned}
& u(r, \theta)=A_{0}+B_{0} \ln r+\sum_{n=1}^{\infty}\left[\left(A_{n} r^{n}+B_{n} r^{-n}\right)\right. \\
& \left.\cos n \theta+\left(C_{n} r^{n}+D_{n} r^{-n}\right) \sin n \theta\right]
\end{aligned}
$$

where the Fourier coefficients $A_{0}, B_{0}, A_{n}, B_{n}, C_{n}$, and $D_{n}$ are found by solving the following six equations [13]:

$$
\begin{gathered}
A_{0}+B_{0} \ln r_{1}=\frac{1}{2 \pi} \int_{0}^{2 \pi} f_{1}(\theta) d \theta \\
A_{n} r_{1}^{n}+B_{n} r_{1}^{-n}=\frac{1}{\pi} \int_{0}^{2 \pi} f_{1}(\theta) \cos n \theta d \theta \\
C_{n} r_{1}^{n}+D_{n} r_{1}^{-n}=\frac{1}{\pi} \int_{0}^{2 \pi} f_{1}(\theta) \sin n \theta d \theta \\
A_{0}+B_{0} \ln r_{2}=\frac{1}{2 \pi} \int_{0}^{2 \pi} f_{2}(\theta) d \theta \\
C_{n} r_{2}^{n}+D_{n} r_{2} r_{2}^{n}+B_{n} r_{2}^{-n}=\frac{1}{\pi} \int_{0}^{2 \pi} f_{0}^{2 \pi} f_{2}(\theta) \sin n \theta d \theta
\end{gathered}
$$

Now we can obtain an analytical solution for the eccentric case by using the conformal mapping.

\section{GEOMETRIC CHARACTERIZATION OF THE BIPOLAR COORDINATE}

Bipolar coordinate $(\xi, \eta)$ is defined by

$$
x+i y=i c \cot \left(\frac{1}{2} \zeta\right), \quad \zeta=\xi+i \eta
$$

where $c>0$. Equation (22) yields

$$
x=c \frac{\sin h \eta}{\cos h \eta-\cos \xi}, \quad y=c \frac{\sin \xi}{\cos h \eta-\cos \xi}
$$

where $-\pi \leq \xi<\pi,-\infty<\eta<\infty$. By eliminating $\xi$ in Equation (23), we obtain a circle with the center of $(c \cot h \eta, 0)$ and the radius of $c \csc h \eta$ as shown below:

$$
(x-c \cot h \eta)^{2}+y^{2}=c^{2} \operatorname{csch}^{2} \eta
$$

Elimination of $\eta$ from Equation (23) results in another circle with the center of $(0, c \cot \xi)$ and the radius of $c \csc \xi$ as given below:

$$
x^{2}+(y-c \cot \xi)^{2}=c^{2} \csc ^{2} \xi
$$

From Equations (24) and (25), the bipolar coordinates are shown in Figure 2. Denoting by $\left(\Gamma_{1}, \varphi_{1}\right)$ and $\left(\Gamma_{2}, \varphi_{2}\right)$, the polar coordinates for the poles of $( \pm c, 0)$, in Figure 3, respectively, we have

$$
\begin{gathered}
x+i y-c=\Gamma_{1} \mathrm{e}^{i \varphi_{1}}, x+i y+c=\Gamma_{2} \mathrm{e}^{i \varphi_{2}} \\
\eta=\log \left(\frac{\Gamma_{2}}{\Gamma_{1}}\right), \xi=\varphi_{1}-\varphi_{2}
\end{gathered}
$$

It follows that a curve $\xi=$ constant is a family of circles passing through the poles $( \pm c, 0)$. The curve of $\eta=$ constant shows a curve for which $\Gamma_{2} /$ $\Gamma_{1}=$ constant. The proof is shown in the Appendix 1. Family of circles are drawn in Figure 2. The outer radius $R_{1}$, inner radius $R_{2}$ and the displacement $b$ are determined by Equation (24) as shown below:

$$
\begin{gathered}
R_{1}=c \operatorname{csch}\left(\eta_{1}\right) \\
R_{2}=c \operatorname{csch}\left(\eta_{2}\right) \\
b=c\left[\operatorname{coth}\left(\eta_{1}\right)-\operatorname{coth}\left(\eta_{2}\right)\right]
\end{gathered}
$$

To describe an eccentric circle in the bipolar coordinate, the three quantities, $c, \eta_{1}$, and $\eta_{2}$ should be determined as shown below:

$$
c=\frac{\sqrt{R_{1}^{4}+R_{2}^{4}-2 R_{1}^{2} R_{2}^{2}-2 b^{2}\left(R_{1}^{2}+R_{2}^{2}\right)+b^{4}}}{2 b}
$$

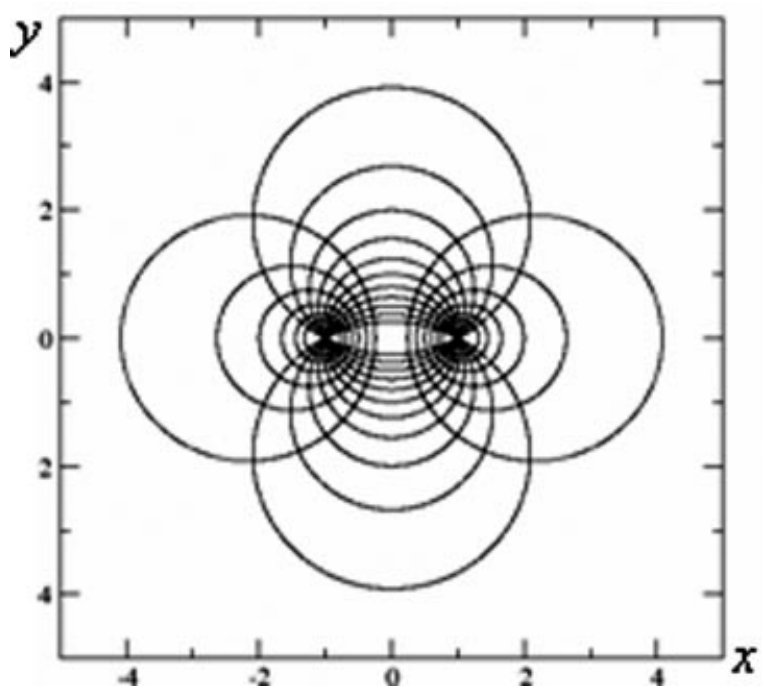

Figure 2 Bipolar coordinate system (poles located on $( \pm 1,0))$. 


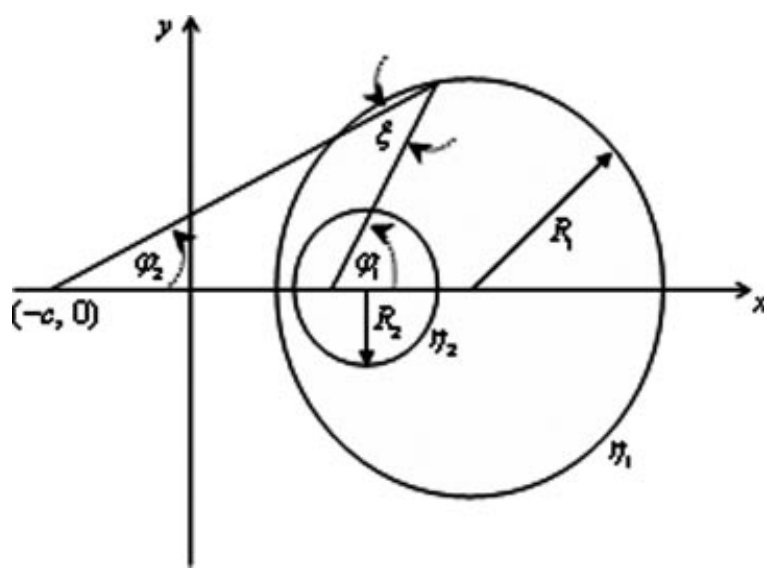

Figure 3 Geometry relation of bipolar coordinate.

$$
\begin{aligned}
& \eta_{1}=\sin h^{-1} \frac{c}{R_{1}} \\
& \eta_{2}=\sin h^{-1} \frac{c}{R_{2}}
\end{aligned}
$$

Then we can describe an eccentric annulus by using the bipolar coordinate.

\section{Viewpoint of Conformal Mapping for the Bipolar Coordinate}

According to the inverse cotangent function [2] as shown below:

$$
\cot ^{-1}(z)=\frac{i}{2} \log \frac{z-i}{z+i}
$$

Equation (22) can be rewritten as:

$$
w(z)=\zeta=i \log \frac{z+c}{z-c}
$$

Equation (35) indicates that the curvilinear bipolar coordinate stems from the conformal mapping of Equation (22). The coordinate $\xi$ changes from $-\pi$ to $\pi$ on crossing the segment of the $x$-axis joining the pole, its range for the whole plane being $-\pi$ to $\pi$. The transformation from Equation (35), where the principal branch of the logarithmic function is used, can be decomposed to two mappings,

$$
\begin{gathered}
Z=\frac{z+c}{z-c} \\
w=\log (Z)
\end{gathered}
$$

More precisely, by expressing $Z=\mathbb{R} \exp (i \Theta)$ we obtain

$$
\log (Z)=\ln \mathbb{R}+i \Theta, \quad(\mathbb{R}>0,-\pi<\Theta<\pi)
$$

We can understand that the point $Z=\mathcal{R} e^{i \Theta_{0}}$ $\left(0<\Theta_{0}<\pi\right)$ moves outward from the origin along the ray $\Theta=\Theta_{0}$, and its transformation is the point whose rectangular coordinate in the $w$ plane is $\left(\ln \mathcal{R}, \Theta_{0}\right)$. That transformation evidently moves to the right along the entire length of the horizontal line $v=\Theta_{0}$. Based on this transformation, we can find that an eccentric annulus can be mapped onto a rectangular domain.

\section{Derivation of an Analytical Solution for Eccentric Problems Using the Bipolar Coordinate}

By using the bipolar coordinate, the eccentric domain can be mapped into the rectangular domain of $(\xi, \eta)$. Note that there are discontinuities in this mapping at $\xi=0$ and $\xi=\pi$. This is due to the symmetry in the geometry above and below the $x$-axis. Since the boundary condition is not necessarily symmetric to $(c, 0)$ about this axis, the problem must be solved piecewise over the interval $0<\xi<\pi$ and $-\pi<\xi<0$. By using this transformation, the solution $f(x, y)=F(\xi, \eta)$ also satisfies the Laplace equation in the curvilinear coordinate as shown below:

$$
F_{\xi \xi}+F_{\eta \eta}=0
$$

The boundary conditions are given below in a general form as:

$$
\begin{array}{ll}
F\left(\eta_{1}, \xi\right)=F_{1}(\xi), & -\pi<\xi<\pi \\
F\left(\eta_{2}, \xi\right)=F_{2}(\xi), & -\pi<\xi<\pi
\end{array}
$$

In addition to the specified boundary conditions, the following natural boundary conditions arise due to the periodic circular symmetry:

$$
\begin{aligned}
& F_{\xi}(\eta, 0)=0 \\
& F_{\xi}(\eta, \pi)=0
\end{aligned}
$$

The solution is obtained by using separation of variables which yields a series solution. This solution is valid on both piecewise intervals and is given by:

$$
\begin{gathered}
F(\eta, \xi)=a_{0}+b_{0} \eta+\sum_{n=1}^{\infty}\left[\left(a_{n} \mathrm{e}^{n \eta}+b_{n} \mathrm{e}^{-n \eta}\right)\right. \\
\left.\cos (n \xi)+\left(c_{n} \mathrm{e}^{n \eta}+d_{n} \mathrm{e}^{-n \eta}\right) \sin (n \xi)\right]
\end{gathered}
$$

where the Fourier coefficients $a_{0}, b_{0}, a_{n}, b_{n}, c_{n}$, and $d_{n}$ are determined by solving the following six equations simultaneously, 


$$
\begin{gathered}
a_{0}+b_{0} \eta_{1}=\frac{1}{2 \pi} \int_{0}^{2 \pi} F_{1}(\xi) d \xi \\
a_{0}+b_{0} \eta_{2}=\frac{1}{2 \pi} \int_{0}^{2 \pi} F_{2}(\xi) d \xi \\
a_{n} \mathrm{e}^{n \eta_{1}}+b_{n} \mathrm{e}^{-n \eta_{1}}=\frac{1}{\pi} \int_{0}^{2 \pi} F_{1}(\xi) \cos (n \xi) d \xi \\
a_{n} \mathrm{e}^{n \eta_{2}}+b_{n} \mathrm{e}^{-n \eta_{2}}=\frac{1}{\pi} \int_{0}^{2 \pi} F_{2}(\xi) \cos (n \xi) d \xi \\
c_{n} \mathrm{e}^{n \eta_{1}}+d_{n} \mathrm{e}^{-n \eta_{1}}=\frac{1}{\pi} \int_{0}^{2 \pi} F_{1}(\xi) \sin (n \xi) d \xi \\
c_{n} \mathrm{e}^{n \eta_{2}}+d_{n} \mathrm{e}^{-n \eta_{2}}=\frac{1}{\pi} \int_{0}^{2 \pi} F_{2}(\xi) \sin (n \xi) d \xi
\end{gathered}
$$

where $F_{1}(\xi)$ and $F_{2}(\xi)$ are the associated boundary conditions given piecewise over the intervals $-\pi<\xi<0$ and $0<\xi<\pi$ as shown in Equations (40) and (41).

\section{AN ILLUSTRATIVE EXAMPLE}

Suppose we want to determine a harmonic function $\psi$ in an eccentric annular region lying between two nonconcentric circles, and satisfying $\psi=0$ on the inner circle with a radius 1 and $\psi=1$ on the outer circle with radius $5 / 2$, where the center of outer circle located on the circumference of inner circle [1]. Several researchers, Carrier and Pearson [1], Muskhelishvili [4], Ling [8], Timoshenko and Goordier [9], Shen [5], Lebedev et al. [11] and Chen and Weng [7] have employed their approaches to solve this problem as shown in Tables 1 and 2. Although the result of Lebedev et al.'s [11] approach using the hyperbolic tangent function is not shown in Tables 1 and 2, it is different from the Timoshenko and Goordier formulation by inversion and rotation only. The geometry description of various formulations using the viewpoint of conformal mapping is shown in Table 1, and their relations are

Table 1 Geometry Transformation of Various Formulations Using the Viewpoint of Conformal Mapping

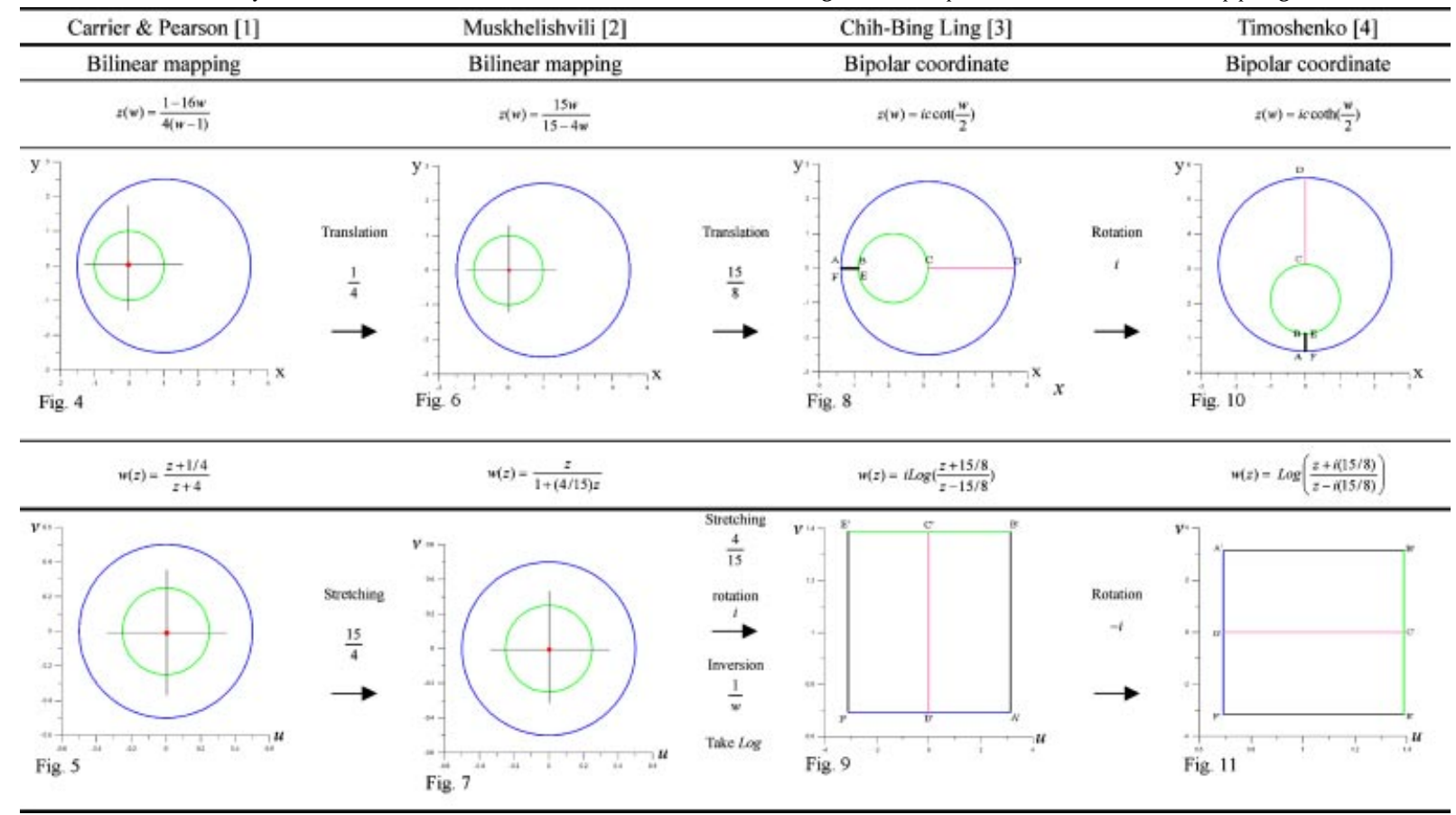

where $z=x+i y$ and $w=u+i v$. 
Table 2 Analytic Solutions of Dirichlet Laplace Problems Using Different Formulations (Blue Curve =1, Green Curve $=0$ )

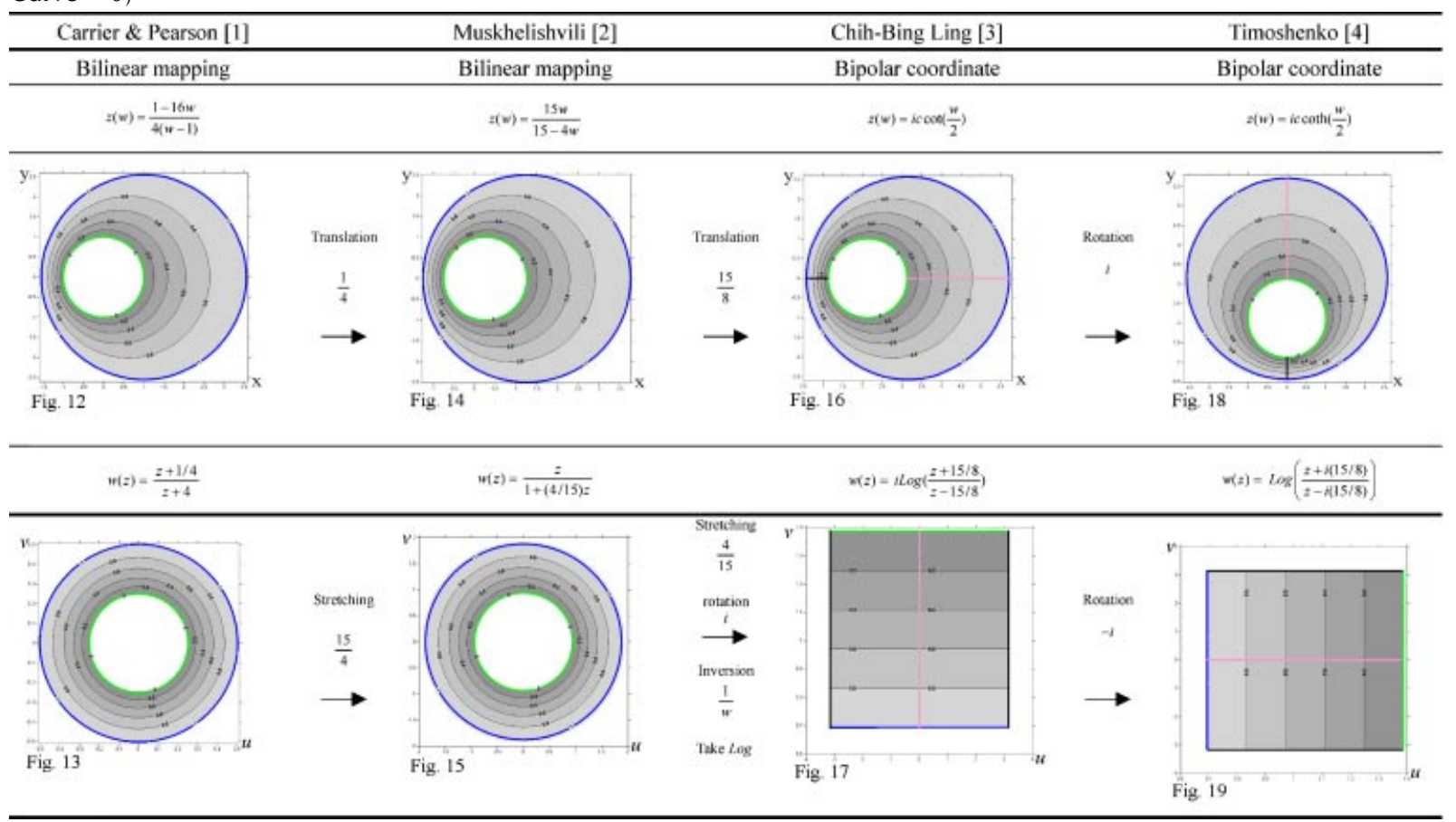

where $z=x+i y$ and $w=u+i v$.

connected through the operations of translation, stretching, rotation, and inversion. We present a case by using four methods in Table 1, and the mapping functions for each method are shown below:

Carrier and Pearson [1]:

$$
w(z)=\frac{z+1 / 4}{z+4}
$$

Muskhelishvili [4]:

$$
w(z)=\frac{z}{1+(4 / 15) z}
$$

Ling [8]:

$$
w(z)=i \log \left(\frac{z+15 / 8}{z-15 / 8}\right)
$$

Timoshenko and Goordier [9]:

$$
w(z)=\log \left(\frac{z+i(15 / 8)}{z-i(15 / 8)}\right)
$$

The eccentric annular problem needs three parameters to describe the geometry, eccentricity e, radii of large and small circles. We set the larger radius to be $5 / 2$ and 1 for the smaller radius with the eccentricity 1 . For different observer systems, we have four geometry descriptions for the same problem. After setting the observer system, each case can be mapped to a regular domain by using equations of (51)-(54), respectively. According to Table 1, Figure 4 can be obtained from Figure 6 by translating 1/4. By translating 15/8 again, we have Figure 8. By multiplying $i$

Table 3 Geometry Description

\begin{tabular}{lccc}
\hline Carrier and Pearson [1] & Muskhelishvili [4] & Ling [8] & Timoshenko and Goordier [9] \\
\hline$\left|z_{g}\right|=1,\left|z_{b}-1\right|=5 / 2$ & $\left|z_{g}-1 / 4\right|=1$, & $\eta_{g}=0.693147$, & $\xi_{g}=0.693147, \xi_{b}=1.38629$, \\
& $\left|z_{b}-5 / 4\right|=5 / 2$ & $\eta_{b}=1.38629, c=15 / 8$, & $c=15 / 8,\left|z_{g}-17 / 8 i\right|=1$, \\
& & $\left|z_{g}-17 / 8\right|=1$, & $\left|z_{b}-25 / 8 i\right|=5 / 2$ \\
$\left|w_{g}\right|=1 / 4,\left|w_{b}\right|=1 / 2$ & $\left|w_{g}\right|=15 / 16,\left|w_{b}\right|=15 / 8$ & $\left|z_{b}-25 / 8\right|=5 / 2$ & $A^{\prime}\left(\pi, \ln \left(\eta_{b}\right)\right), B^{\prime}\left(\pi, \ln \left(\eta_{g}\right)\right)$, \\
& & $E^{\prime}\left(\ln \left(\eta_{g}\right), \pi\right), F^{\prime}\left(\ln \left(\eta_{b}\right), \pi\right)$, & $\left.E^{\prime}\left(\pi, \ln \left(\xi_{b}\right), \pi\right), B^{\prime}\left(\ln \left(\xi_{g}\right)\right), \pi\right), F^{\prime}\left(\pi, \ln \left(\xi_{b}\right)\right)$ \\
\hline
\end{tabular}




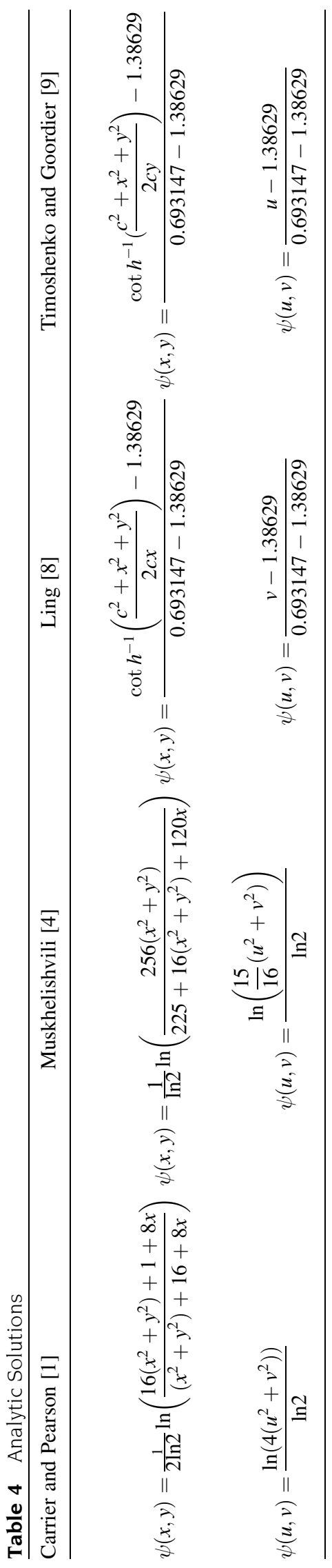

(rotation of $90^{\circ}$ ) of Figure 8, we obtain Figure 10. By stretching 15/4 of Figure 5, we have Figure 7. By stretching $4 / 15$, rotation $i$, inversion $1 / w$ and taking $\log (w)$ of Figure 7, we transform to a rectangular domain of Figure 9. By multiplying $-i$ (counterclockwise rotation of $90^{\circ}$ ), Figure 11 can be obtained from Figure 9. Finally, it can be unified together through the operations of stretching, translation, rotation, inversion and taking Log. Analytic solutions are shown in Table 2 using various formulations and the contour plots are also shown to compare with each other. Table 3 shows the geometry description while Table 4 indicates the closed-form solution.

\section{CONCLUSIONS}

In this paper, various approaches including Carrier and Pearson [1], Muskhelishvili [4], Ling [8], Timoshenko and Goordier [9], and Lebedev et al. [11] for solving eccentric Laplace problems were reviewed. Based on the conformal mapping, all of them are unified together. The relations among them are constructed through the operations of translation, rotation, and inversion. One example was demonstrated to understand the relationship among various formulations.

\section{APPENDIX}

From Equation (22), we have

$$
z=-c \operatorname{coth}(i \zeta)=-c \frac{\cos h(i \zeta)}{\sin h(i \zeta)}=-c \frac{\mathrm{e}^{i \zeta}+\mathrm{e}^{-i \zeta}}{\mathrm{e}^{i \zeta}-\mathrm{e}^{-i \zeta}}
$$

where $\zeta=((\xi+i \eta) / 2)$. Equation (A1) yields

$$
\begin{aligned}
\left(\mathrm{e}^{i \zeta}-\mathrm{e}^{-i \zeta}\right) z & =-c\left(\mathrm{e}^{i \zeta}+\mathrm{e}^{-i \zeta}\right) \\
(z+c) \mathrm{e}^{i \zeta} & =(z-c) \mathrm{e}^{-i \zeta} \\
\mathrm{e}^{2 i \zeta} & =\frac{z-c}{z+c}
\end{aligned}
$$

since $z-c=\Gamma_{1} \mathrm{e}^{i \varphi_{1}}$ and $z+c=\Gamma_{2} \mathrm{e}^{i \varphi_{2}}$

$$
2 i \zeta=\log \frac{\Gamma_{1} \mathrm{e}^{i \varphi_{1}}}{\Gamma_{2} \mathrm{e}^{i \varphi_{2}}} .
$$

According to the definition of $\zeta=((\xi+i \eta) / 2)$, we obtain

$$
i \xi-\eta=\log \frac{\Gamma_{1}}{\Gamma_{2}}+i\left(\varphi_{1}-\varphi_{2}\right)
$$

Therefore, the equations of real and imaginary parts yield 


$$
\eta=\log \frac{\Gamma_{2}}{\Gamma_{1}}, \quad \xi=\varphi_{1}-\varphi_{2}
$$

This concludes the proof that $\left(\Gamma_{1} / \Gamma_{2}\right)$ does not change for the constant $\eta$ as shown in Figure 3.

\section{REFERENCES}

[1] G. F. Carrier and C. E. Pearson, Partial differential equation-theory and technique. New York, Academic Press, 1976, pp 68-71.

[2] J. W. Brown and R. V. Churchill, Complex variables and applications. New York, McGraw-Hill, 2003.

[3] O. E. Lafe, J. S. Montes, A. H. D. Cheng, J. A. Liggett, and P. L.-F. Liu, Singularity in Darcy flow through porous media, J Hydraulic Div, 106 (1980), 977997.

[4] N. I. Muskhelishvili, Some basic problems of the mathematical theory of elasticity. Noordhoff, Groningen, 1953, pp 175-179.

[5] W. C. Shen, "Null-field approach for Laplace problems with circular boundaries using degenerate

\section{BIOGRAPHIES}

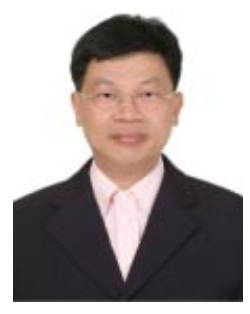

Jeng-Tzong Chen, born in 1962, received a BS degree in Civil Engineering, an MS in Applied Mechanics, and a $\mathrm{PhD}$ in Civil Engineering, respectively, in 1984, 1986, and 1994, from National Taiwan University, Taipei, Taiwan, R.O.C. He had worked as a research assistant in the Structural Division of the Department of Rocket and Missile System, Chung Shan Institute of Science and Technology, from 1986 to 1990 . In 1994, he was invited to be an Associate Professor in the Department of Harbor and River Engineering, National Taiwan Ocean University, Keelung, Taiwan, R.O.C. He was promoted to full professor in 1998. Later in 2004, he was selected to be the distinguished professor. Furthermore, he was selected as the life-time distinguished professor in 2007. His major interest is computational mechanics. He had derived the theory of dual integral equations for boundary value problems with degenerate boundary. Prof. Chen also developed four dual BEM programs for the BVPs of Laplace equation, Helmholtz equation, bi-Helmholtz and modified Helmholtz equation and Navier equation. Recently, he also employed the null field integral equations to solve BVPs with circular boundaries. He wrote two books in Chinese on dual BEM and FEM using MSC/NASTRAN, respectively. He was ever invited to give plenary and keynote lectures, for example, twice in World Congress on Computational Mechanics (WCCM4 in Buenos Aries and WCCM5 in Vienna) and FEM/BEM 2003 in St. Petersburg, Russia. Also, he is the editor of Journal of the Chinese Institute of Civil and Hydraulic Engineering. $\mathrm{He}$ has been the Editor of Journal of Marine Science and Technology and the guest editor of J. Chinese Institute of Engineers. $\mathrm{He}$ won several times of Outstanding Research Awards from kernels", Master Thesis, National Taiwan Ocean University, Keelung, Taiwan, 2005.

[6] M. R. Spiegel, Theory and problems of complex variables. New York, McGraw-Hill, 1981.

[7] T. Chen and I. S. Weng, Torsion of a circular compound bar with imperfect Interface, J Appl Mech, 68 (2001), 955-958.

[8] C. B. Ling, "Torsion of an eccentric circular tube", Technical Report, No. 1, Chinese Bureau of Aeronautical Research, 1940.

[9] S. P. Timoshenko and J. N. Goordier, Theory of elasticity. New York, McGraw-Hill, 1972, pp 196-202.

[10] A. F. Bearson, Complex analysis: The argument principle in analysis and topology. New York, Wiley, 1989.

[11] N. N. Lebedev, I. P. Skalskaya, and Y. S. Uflyand, Worked problems in applied mathematics. New York, Dover, 1965.

[12] L. S. Stephens and M. A. Casemore, Negative stiffness coefficients for magnetic actuators using Laplace's equation, J Eng Gas Turbines Power, 123 (2001), 612-618.

[13] S. J. Farlow, Partial differential equations for scientists and engineers. Canada, Wiley, 1982.
National Science Council, Taiwan. He also won the Wu, Ta-You Memorial Award in 2002. He is currently the member of editorial board of five international journals. Until now, he has published more than 125 SCI papers on BEM and FEM in technical journals. Nearly 582 citings can be found. Boundary element method is one focus of Professor Chen's research interests. Others may be categorized into three areas. One is vibration and acoustics, another is computational mechanics and the other is engineering and mathematical education. E-mail: jtchen@mail.ntou.edu.tw; URL: http://ind.ntou.edu.tw/ msvlab.

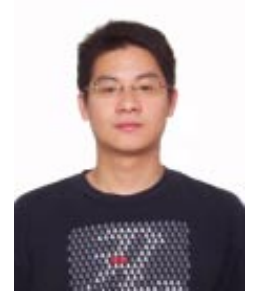

Ming-Hong Tsai was formerly an undergraduate student in the Department of Harbor and River Engineering at National Taiwan Ocean University. He is currently a graduate student in the Department of Engineering Science and Ocean Engineering at National Taiwan University.

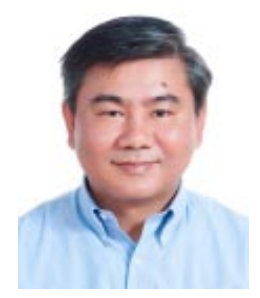

Chein-Shan Liu was born in Taiwan on May 21, 1958. Since August 2000, he has been a faculty member of the Department of Mechanical and Mechatronic Engineering at National Taiwan Ocean University. His research interests can be categorized into five areas: material characterization, nonlinear dynamics, inverse problem, numerical method, applied mathematics. 